\title{
Performance Analysis of Mobile Ad Hoc Unmanned Aerial Vehicle Communication Networks with Directional Antennas
}

\author{
Abdel Ilah Alshbatat ${ }^{1}$ and Liang Dong ${ }^{2}$ \\ ${ }^{1}$ Department of Electrical Engineering, Tafila Technical University, Tafila 66110, Jordan \\ ${ }^{2}$ Department of Electrical and Computer Engineering, Western Michigan University, Kalamazoo, MI 49008, USA
}

Correspondence should be addressed to Abdel Ilah Alshbatat, a.alshabatat@ttu.edu.jo

Received 8 April 2010; Revised 5 October 2010; Accepted 31 December 2010

Academic Editor: Hyochoong Bang

Copyright () 2010 A. I. Alshbatat and L. Dong. This is an open access article distributed under the Creative Commons Attribution License, which permits unrestricted use, distribution, and reproduction in any medium, provided the original work is properly cited.

Unmanned aerial vehicles (UAVs) have the potential of creating an ad hoc communication network in the air. Most UAVs used in communication networks are equipped with wireless transceivers using omnidirectional antennas. In this paper, we consider a collection of UAVs that communicate through wireless links as a mobile ad-hoc network using directional antennas. The network design goal is to maximize the throughput and minimize the end-to-end delay. In this respect, we propose a new medium access control protocol for a network of UAVs with directional antennas. We analyze the communication channel between the UAVs and the effect of aircraft attitude on the network performance. Using the optimized network engineering tool (OPNET), we compare our protocol with the IEEE 802.11 protocol for omnidirectional antennas. The simulation results show performance improvement in end-to-end delay as well as throughput.

\section{Introduction}

Mobile ad hoc network (MANET) is a wireless network that is formed by a collection of self-organizing mobile nodes. Each node communicates with its neighbors over a shared wireless medium. Due to the lack of central management, nodes in MANET are designed to act as end systems and routers for other nodes. The network is connected dynamically and does not rely on any pre-existing network infrastructure. In MANET, nodes are free to move and have the capability to deliver messages in a decentralized manner. A major challenge is how to route data packets over such network that changes its structure dynamically due to member mobility, especially when the source and the destination are out of transmission range [1].

Recently, there has been an increasing interest in employing unmanned aerial vehicle (UAV) in wireless communication networks [2]. UAV has been used in military applications as well as civilian. It shows great advantages in search and rescue, real-time surveillance, reconnaissance operations, traffic monitoring, and range extension [3]. With the advent of low-cost commercial off-the-shelf wireless communication equipment embedded in the UAV platform, a swarm of UAVs can form a MANET in the air. UAV ad hoc communication network is a new type of wireless network for communication among multiple UAVs. A collection of autonomous UAVs dynamically form a temporary multihop radio network without the aid of any centralized station.

In UAV MANET communication environments, due to the mobility of nodes, network topology may change rapidly and unpredictably. As a result, nodes are expected to act cooperatively to establish network connection and to route data packets over multiple hops for long distances [4]. A network of low-altitude UAVs is usually complex compared with other types of wireless networks [5]. Wireless link created by UAVs may alter in link quality over time due to a number of factors such as Doppler effects, changes in communication distance, and blocking of line-of-sight by the aircraft body. One challenge to use UAV as a node in MANET is the effect of aircraft attitude on the wireless link quality. The impact of aircraft attitude (pitch, roll, and yaw) on the MANET performance is significant. In particular, aircraft attitude affects the end-to-end delay and the throughput. These effects increase the retransmissions overhead and thus 
reduce the overall throughput and increase the end-to-end delay. In order to reduce the impact of aircraft attitude, there is a need for designing a medium access control (MAC) protocol for such communication system that will compensate for these effects.

Using miniature UAVs in wireless communication networks may introduce a new challenge. The size of the lightweighted UAV is so small when compared with the size of communication equipment required to transmit data over long distances. One solution is to use high-gain directional antennas. Directional antennas provide a number of advantages over omnidirectional antennas. As its name implies, directional antenna allows the signal to be transmitted in one direction more efficiently, and at the same time cutting down the interference by ignoring signals coming from places other than the desired one. Moreover, directional antenna is being recognized nowadays as a powerful method for increasing the connectivity of ad hoc networks. The transmission range of directional antennas is usually larger than that of omnidirectional antennas, resulting in the reduction in hops between source and destination. Meanwhile, there is a need to develop efficient distributed algorithms to cope with the aircraft dynamics that affects the directivity of directional antennas.

In this paper, we consider a collection of UAVs that communicate through wireless links as a MANET using directional antennas. All UAVs including miniature ones are equipped with unity gain omnidirectional antennas and directional antennas. Because of the size and power limitations, the use of the directional antennas in this paper is to increase the quality and reliability of the communication link without adding additional equipment, complexity, and power consumption to the UAVs. Current MAC protocol (IEEE 802.11) that implements omnidirectional antennas may not be suitable while using directional antennas. Therefore, there is a need for designing a new directional MAC protocol that is capable for adapting any constraints imposed by the UAV MANET. In that respect, to integrate the directional antenna successfully into UAV ad hoc networks and to realize its benefits within the MAC layer [6-10] and the network layer, a new MAC protocol is introduced in this paper so that the use of directional antenna will not become a problem for the routing protocol but indeed will enhance the performance of the network.

The remainder of this paper is organized as follows. In Section 2, we give a survey of current research regarding the directional MAC protocols and the concept of using UAV as a communication node in MANET. In Section 3, we describe our scheme which is called Adaptive Medium Access Control Protocol for Unmanned Aerial Vehicle (AMAC_UAV) and discuss its capability of adapting to the external parameters imposed by the UAV. In Section 4 , we provide a statistical model for the wireless channel between two UAVs and the packet transmission time. In Section 5, we explain our implementation of the UAV communication network in OPNET, and in Section 6, we present the simulation results and provide a comparison with the MAC protocol for omnidirectional antennas (IEEE 802.11). Finally, we summarize the paper in Section 7.

\section{Related Work}

Recently, a large number of MAC schemes have been proposed for MANETs that are equipped with directional antennas. In general, most schemes that discussed the directional antennas are focused on the modification of the MAC protocols [11-14]. Some researchers have suggested the use of switched beam antenna while others suggested the use of adaptive antenna. Nasipuri et al. [15] proposed a directional MAC protocol that utilizes switched beam antenna. They showed that by using four directional antennas, the average throughput of the network could be improved up to three times over that of using omnidirectional antenna. They assumed that the gain of the directional antenna is equal to the gain of the omnidirectional antenna. In their mechanism, the transmissions and receptions involve omnidirectional antenna. The complete cycle starts by sending RequestTo-Send (RTS) packet using the omnidirectional antenna. Receiver responds with a Clear-To-Send (CTS) packet also using the omnidirectional antenna. As soon as the transmitter receives the CTS packet, it estimates the angle of arrival (AOA) of this packet and transmits data using the directional antenna.

In [7], the authors assumed (as in [15]) that the directional gain equals the omnidirectional gain and proposed two MAC schemes. In the first scheme, RTS, acknowledgment $(\mathrm{ACK})$ and data packets are sent directionally while CTS packet is sent omnidirectionally. Other nodes that hear the CTS should block the antenna on which it was received. In the second scheme, they proposed two types of RTS, Directional Request-To-Send (DRTS) and Omnidirectional Request-To-Send (ORTS) based on the following rule: (1) if none of the directional antennas of the node is blocked, the node will send ORTS. (2) Otherwise, the node will send a DRTS provided that the desired directional antenna is not blocked. The CTS, DATA, and ACK packets are the same as in the first scheme. Compared with the scheme in [15], the node according to the MAC scheme in [7] may transmit in directions that do not interfere with the ongoing transmissions.

Other researchers [16-18] studied the performance of MAC protocols with adaptive antenna array. Bao, et al. [18] developed a distributed Receiver-Oriented Multiple Access (ROMA) protocol for ad hoc networks in which all nodes are equipped with a multibeam adaptive antenna array. ROMA is capable of forming multiple beams and creating several simultaneous communication sessions. Another scheme was developed by the authors, called Neighbor-Tracking, which is used to schedule transmissions by each node in a distributed way.

A caching mechanism is a new technique which was proposed to facilitate the operation of the MAC protocol for a node that is equipped with directional antenna [19]. A new carrier sensing mechanism called Directional Virtual Carrier Sensing (DVCS) was presented in [19]. This mechanism needs information about AOA for each signal from the physical layer. The authors have proposed the use of a caching mechanism to store information about angular location of neighboring nodes. Whenever the MAC layer 
receives a packet from the upper layer, it will look in the cache to determine whether it has the information about the angular position of the destination node. If the angular position of the destination node is known, the packet is transmitted using the directional antenna, otherwise it will be sent using the omnidirectional antenna.

The authors in [20] designed another MAC protocol which uses multihop RTS to establish links between distant nodes; they called their protocol MMAC. In MMAC, when any node receives RTS, it transmits CTS, DATA, and ACK over a single hop. In $[19,20]$, it was suggested to use the Directional Network Allocation Vector (DNAV). DNAV is similar to the NAV that is used in standard IEEE 802.11 except that the DNAV stores the angle of arrival of the RTS packets in any given direction. For each packet to be transmitted, the DNAV is consulted to see whether the angle of the packet to be transmitted is overlapped with any ongoing transmissions. If there are overlaps, the packet transmission is deferred; otherwise, the packet is transmitted.

In [21], the authors presented a scheme called utilizing directional antennas for ad hoc network (UDAAN). UDAAN consists of several new mechanisms such as neighbor discovery with beam forming, link characterization for directional antennas, and proactive routing and forwarding. They have shown that employing directional antennas improves system performance.

Orientation handoff is another name for the mechanism that is created while integrating directional antenna with MAC protocol. This technique was invented to describe the process of switching from omnidirectional transmission to directional transmission. In [22], the authors proposed a novel preventive link maintenance scheme based on directional antennas. They aimed to extend the life of the link that is about to break. A warning is generated within a node when the received power is reduced below a certain threshold. A node then switches to the process of creating a directional antenna pattern to raise the received power so that the link will not break.

Although directional antennas offer many benefits to MANET, they also present new problems. In [23], the author proposed a new mechanism to solve different problems using directional antenna, for instance, hidden terminal problem and exposed terminal problem. All these problems are solved by building a MAC timing structure. In [24], the authors analyzed the performance of a wireless network using directional antenna based on a different coding scheme. In addition, they analyzed the effect of direction estimation error on the network performance. They derived the cumulative distribution function of the signal-to-interference-andnoise ratio (SINR) for a certain link and then analyzed the outage probability of that link.

Locating and tracking nodes under mobility is a challenge in ad hoc network. In most of the previous work, the authors assumed that the transmitter knows the receiver's location. This assumption may not be true due to the fact that offering nodes' positions may increase the overhead packets, thus the MAC protocol should offer a mechanism to locate and track node neighbors. Korakis et al. [25] proposed the use of a circular RTS (CRTS) message to solve this problem. In their protocol, RTS/CTS packets are transmitted on every beam. By doing so, they achieved a higher range but at the cost of high control overhead.

In [26], the authors proposed a polling-based MAC protocol that addresses the problem of neighbor discovery in the use of directional antennas. The proposed MAC protocol is based on the polling strategy wherein a node polls its neighbors periodically. Time is segmented into consecutive frames and nodes are synchronized with each other. By this technique, each node is able to adjust its antenna weight in order to track its neighbors.

Deafness problem is another challenge to ad hoc network with directional antennas. This problem is created as a result of exchanging RTS/CTS directionally. In [27], the authors proposed a new protocol called Toned MAC. Deafness problem was addressed by using subband tones. Tones are sinusoidal signals that do not contain information bits and thus do not require demodulation. They are only detected through energy estimation and thus notify the neighbors of a communicating node. The channel in a node that implements this protocol must be divided in two subchannels: the data channel and the control channel. The data channel is used for transmitting the four-way handshaking while the control channel is used for transmitting the tone signal. Each tone-frequency is identified by a unique code to assist nodes in determining the sender of a given tone.

In [28], the authors proposed a MAC protocol called Adaptive Beam-Forming Carrier Sense Multiple Access/Collision Avoidance (ABF-CSMA/CA) by using smart antenna. This protocol, as others, employs the RTS/CTS/DATA/ACK access mechanism to manage node communications. In this protocol, training sequences are transmitted before applying directional RTS and CTS packets. Training sequences are mainly used to estimate the behavior of the wireless channel.

Integrating wireless equipment into a small UAV has been studied recently, especially in the context of MANET where communication is required between nodes that would not be able to communicate because of line-of-site obstructions. In [29], the authors showed that by integrating small low-cost commercial off-the-shelf $802.11 \mathrm{~b}$ equipment into a UAV, a powerful networking node can be created in the air. They also showed that UAVs provided shorter routes that had better throughput than a similar ground-based network. To understand the performance of such a network, the authors in [30] built a wireless network test bed using IEEE 802.11b. The test bed gave detailed data on network throughput, delay, range, and connectivity under different operating regimes.

In [31], the authors addressed the issue of configuring 802.11a antennas in UAV-based networking and presented a set of field experiments (test bed) to the wireless link between UAVs and ground station. They measured the linklayer throughput based on various antenna orientations and communication distances. They concluded that both the UAV and the ground station should use omnidirectional dipole antennas to get high throughput. In addition, they showed that the path loss in an airfield environment is roughly proportional to the square of the communication distance. 
To improve the range and the reliability of ad hoc ground-based networks, the concept of using UAV as a communication relay was presented in [32]. The authors studied the performance of the ad hoc ground network using UAV as a relay node and the effects of UAVs' positions and velocities on Bit-Error-Rate (BER). In [33], the authors presented the Load-Carry-And-Deliver (LCAD) networking paradigm to relay messages between two distant ground nodes. This paradigm, LCAD, is designed for maximizing the throughput of UAV-relaying networks by having a UAV load from a source ground node, carrying the data while flying to the destination, and finally delivering the data to a destination ground node. They compared their paradigm against the conventional multihop and claimed that the proposed LCAD paradigm can be used to provide high throughput between ground nodes.

In spite of these previous efforts, there are still problems that arise with the deployment of directional antennas for UAV communications and these are little work done that handles multiple UAVs that form a MANET in the air. None of the previous approaches considers the effect of aircraft dynamics while implementing directional antennas. Aircraft dynamics is represented by three parameters: pitch, yaw, and roll. Any variation in these parameters could lead to an intermittent channel between the sender and the receiver and impose a significant problem for the UAV communication network.

\section{Adaptive MAC Protocol for Unmanned Aerial Vehicle (AMAC_UAV)}

UAVs are desired nowadays to extend the coverage of communication networks. We propose the use of directional antenna onboard UAV that can be steered in any direction. In contrast to the traditional use of omnidirectional antenna, our approach provides low interference and robustness to the wireless link and provides better network performance in end-to-end delay and throughput. In general, directional antennas can be classified into two types: adaptive array and switched beam antenna. An adaptive array can be implemented with an array of antennas, while a switched beam antenna can apply basic switching between predefined beams. Adaptive array can be more precise than switched beam antenna but with higher complexity in practice.

The performance of the UAV ad hoc network depends on several physical factors such as aircraft mobility and attitude. We assume that all UAVs are off the ground and fly at different altitudes. The distance between any two UAVs will not go beyond the transmission range of the directional antenna. Two pieces of additional hardware are needed in our scheme for UAV localization: Global Positioning System (GPS) and Inertial Measurement Unit (IMU). When a packet arrives from the upper layer, the node requires the position of the destination in order to steer the main lobe in the right direction. Control packet of RTS will be sent using omnidirectional antenna. It includes the position of the aircraft and the duration of transmission. The destination node will respond with a CTS packet that has the same information regarding itself. Each node that hears the CTS or RTS should cache this information and updates its table for future use. The data packet will be sent using directional antenna.

Figure 1 shows the flowchart of the proposed adaptive MAC protocol for UAV ad hoc communication networks with directional antennas. The implementation of this scheme emphasizes the following issues.

(i) Every UAV is equipped with four antennas. Two of these are directional. One directional antenna is located on top of the UAV and marked primary, and the other is located beneath the UAV and marked secondary. The other two antennas are omnidirectional. If the UAV has no packet to send, it will listen to other UAVs using one of the omnidirectional antennas. If the UAV has packets to send, it has the choice to send using either one of the directional antennas or the omnidirectional antennas.

(ii) The locations of the UAVs are important factors in the proposed scheme. With the proposed MAC protocol, each UAV frequently monitors the positions of other UAVs and computes the effect of Euler Angles on the directional antenna.

(iii) The proposed MAC protocol frequently monitors the UAV distance, bit error rate, and retransmit counter so that it switches to omnidirectional antenna if the values exceed the limits.

(iv) In the case that there is no activity during a second, the UAV sends a heartbeat message using the omnidirectional antenna. This message contains the location of the UAV. When it is received by another UAV, the UAV updates its table and responds with a similar heartbeat message.

(v) In the proposed scheme, each UAV is capable of electronically steering the antenna beam toward a specific direction. Our modeling of the antenna is based on a single beam that can target the boresight to any direction.

(vi) In the case that the aircraft changes its attitude, the pattern of the antenna will rotate with respect to its axis, resulting in fluctuations in antenna gain. These fluctuations affect the transmission range of the UAV communication. Therefore, the proposed MAC protocol compensates the antenna gain for any changes by applying the same value to the target location.

(vii) Switching time between primary and secondary directional antennas is assumed negligible.

According to the IEEE 802.11 MAC protocol, a packet is discarded after the retransmit counter exceeds 7. Meanwhile, as the number of retransmission attempts increases, the possibility of delay increases. Based on the Distribution Coordination Function (DCF), a node senses the channel to determine whether it is idle or not. Sensing is done through physical and virtual mechanisms. If the medium is sensed idle for a DCF inter-frame-space (DIFS) interval, 


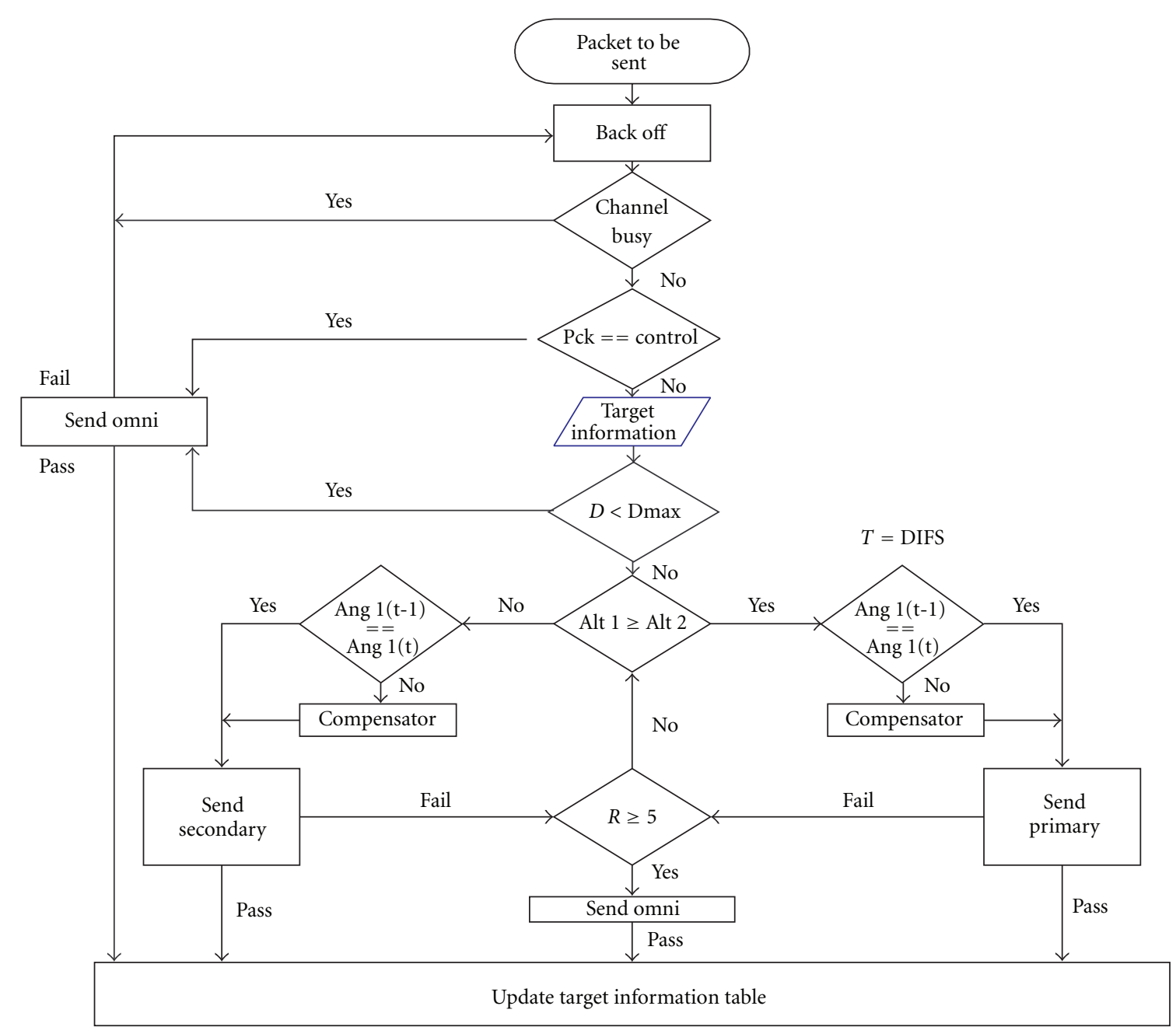

FIGURE 1: Flowchart of MAC protocol for UAV ad hoc communication network with directional antennas.

the node has the right to use the medium and start sending data. On the other hand, if the medium is busy or it becomes busy during the DIFS time interval, the transmission will be deferred for a certain time until no other node occupies the medium. In such situation the backoff timer is enabled. Our scheme follows the IEEE 802.11 standard with some modification to the retransmit counter (retransmission threshold is set to be 5).

There are two methods for carrier sensing in the IEEE 802.11 standard, physical carrier sensing and virtual carrier sensing. Virtual sensing is done through the use of network allocation vector (NAV). Two messages, RTS and CTS, precede the data transmission. These messages contain the duration for which the UAV should reserve the channel to complete the data transmission. On the other hand, any UAV that overhears these messages should defer data transmission for this duration to avoid interference with other UAVs' transmission. In the proposed scheme, the RTS and CTS contain the location and orientation of the UAV. We use the directional network allocation vector (DNAV) mechanism [20] with some modification to adapt our scheme while using directional antennas for UAV communications. Our
DNAV is synchronized with the target information table that is created through the handling of the control messages. In addition, the original NAV is also used in our scheme.

The procedure of the proposed MAC scheme is as follows.

(1) To resolve the hidden terminal problem, the RTS and CTS are exchanged between the UAVs. Consider the case when UAV number one attempts to send a packet to UAV number two. UAV number one will perform physical carrier sensing as in the IEEE 802.11 standard. If the channel is idle, another sensing will be done for NAV to see if the channel is reserved by another UAV. Once the medium as well as the NAV is idle, UAV number one enters the backoff period for a certain time then RTS packet is sent through the omnidirectional antenna along with the parameters of UAV number one (location and orientation).

(2) UAV number one, as well as UAV number two, is equipped with GPS and IMU to provide the position at high rates. Once UAV number one receives RTS from UAV Number One, it senses the channel for 
short-inter-frame-space (SIFS) interval. If the channel is free, it sends CTS along with the its parameters in response using the omnidirectional antenna and updates the target information table as shown in Table 1. Other UAVs that also receive either RTS or CTS update their target information table as well as the DNAV and NAV.

(3) Once UAV number one receives the CTS message, it updates its target information table. Before initiating transmission of the data packets, the MAC checks the distance between the two UAVs. If the distance is less than the transmission range of the omnidirectional antenna (Dmax), the data are sent using the omnidirectional antenna. Otherwise, the MAC checks the UAVs' altitudes. If the altitude of UAV number one is equal or less than that of UAV number one, data are sent through the primary antenna (directional antenna) along with UAV parameters. Antenna beam is steered to the direction of UAV number one. Otherwise, secondary antenna is used and is steered to UAV number two.

(4) As soon as UAV number one receives the data successfully and updates its target information table, ACK is sent using the omnidirectional antenna along with UAV parameters.

(5) For each data packet, the directional antenna is steered based on the destination location as well as the source Euler Angles. To be more specific, consider UAV Number one's attempt to send the second data packet. The location of UAV number one is obtained from the ACK. If UAV number one senses change in the angles after receiving the target location, the MAC compensates for this change by applying the same antenna gain value to the target location.

(6) As mentioned before, a packet is discarded after the retransmit counter exceeds 7 . Since our goal is to minimize the end-to-end delay, the proposed scheme will switch the transmission from directional to omnidirectional if the retransmit counter reaches 5 .

\section{Performance Analysis}

The performance of UAV ad hoc networks shall be measured in terms of achievable throughput and end-to-end delay. Other features can also be investigated to evaluate the robustness of the proposed scheme, such as signal-to-noise ratio (SNR) and bit error rate (BER). In the following, we model the wireless channel between two UAVs statistically and investigate the packet transmission time.

4.1. Statistical Model for Wireless Channel between Two UAVs. To analyze the wireless communication link between two UAV s using directional antennas, let us consider the example in Figure 2. The transmitting UAV $i$ is located at $\left(X_{i}, Y_{i}, Z_{i}\right)$ (center) while the receiving $\mathrm{UAV} j$ at $\left(X_{j}, Y_{j}, Z_{j}\right)$ (left lower). The aspect angle $\Phi$ defines the radiation of UAV $i$ 's antenna with respect to UAV $j$. This angle consists of two parts, the
TABLE 1: Target information table.

\begin{tabular}{lcclc}
\hline $\begin{array}{l}\text { Target } \\
\text { ID }\end{array}$ & $\begin{array}{c}\text { Latitude } \\
\text { (Deg min } \\
\text { sec) }\end{array}$ & $\begin{array}{c}\text { Longitude } \\
\text { (Deg min } \\
\text { sec) }\end{array}$ & $\begin{array}{l}\text { Altitude } \\
\text { (Feet) }\end{array}$ & Direction \\
\hline 1 & $431632 \mathrm{~N}$ & $853846 \mathrm{~W}$ & 500 & 90 \\
2 & $431632 \mathrm{~N}$ & $853836 \mathrm{~W}$ & 450 & 90 \\
\hline
\end{tabular}

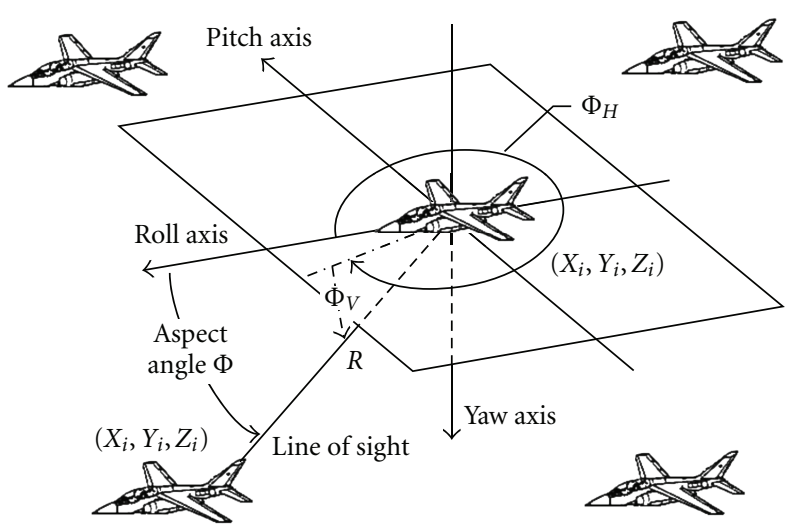

Figure 2: Aspect angle $\Phi$, the angle between the roll and the line of sight.

horizontal aspect angle $\Phi_{H}$ and the vertical aspect angle $\Phi_{V}$. $\Phi_{H}$ is determined by the angle between the roll axis of UAV $i$ and the line of sight (LOS) projected onto the yaw plane of UAV $i$. $\Phi_{V}$ is determined by the angle between the LOS and the projection of the LOS onto the yaw plane of UAV $i$. The above angles depend on the locations of UAV $i$ and UAV $j$ as well as the attitude of UAV $i$.

We assume that each UAV is equipped with a transceiver, directional antennas and omnidirectional antennas. The wireless link between the two UAVs can be modeled with path loss and fast fading. Path loss is basically caused by the distance attenuation of the radiated power, and fading is due to multipath effect of low altitude UAV propagation. Both cases are affected by the high mobility of the UAVs such that there is rapid variation in the received signal power. Moreover, the fading level changes frequently. We assume that there is a clear LOS between the UAVs. The average power of the received signal can be given using the Friis freespace transmission equation.

$$
P_{r}=P_{t} G_{t} G_{r}\left(\frac{\lambda}{4 \pi R}\right)^{2},
$$

where $P_{r}$ represents power received by the receiving antenna and $P_{t}$ power input to the transmitting antenna. $G_{t}$ and $G_{r}$ are the antenna gain of the transmitting and receiving antennas, respectively. $\lambda$ is the wavelength, $R$ is the distance between the two UAVs, and $4 \pi R / \lambda$ is the so-called free-space path loss. We assume that the bandwidth is narrow enough that the wavelength has a single value.

We set the receiving antenna gain $G_{r}\left(\Phi_{H}^{\prime}, \Phi_{V}^{\prime}\right)=1$, where $\Phi_{H}^{\prime}$ and $\Phi_{V}^{\prime}$ are the aspect angels of the receiving UAV $j$ 
toward the transmitting UAV $i$. The received power can be given as

$$
P_{r}=P_{t} G_{t}\left(\Phi_{H}, \Phi_{V}\right)\left(\frac{\lambda}{4 \pi R}\right)^{2} .
$$

Taking logarithm of (2), we have

$$
\begin{aligned}
10 \log _{10}\left(P_{r}\right)= & 10 \log _{10}\left(P_{t}\right)+10 \log _{10}\left(G_{t}\left(\Phi_{H}, \Phi_{V}\right)\right) \\
& -20 \log _{10}\left(\frac{4 \pi R}{\lambda}\right), \\
P_{r}\left(d B_{w}\right)= & P_{t}\left(d B_{w}\right)+G_{t}\left(d B_{i}\right)-Q_{0}(d B),
\end{aligned}
$$

where $Q_{0}$ represents the free-space path loss and is give by

$$
\begin{aligned}
Q_{0}(d B) & =20 \log _{10}\left(\frac{4 \pi R}{\lambda}\right) \\
& =32.4+20 \log _{10}\left(f_{\mathrm{MHz}}\right)+20 \log _{10}\left(d_{\mathrm{km}}\right),
\end{aligned}
$$

where $f_{\mathrm{MHz}}$ is the transmission frequency in $\mathrm{MHz}$ and $d_{\mathrm{km}}$ is the distance between the two UAVs in kilometer.

The received signal in UAV communication experiences random variation due to aircraft mobility, blocking and reflection of the fuselage, and terrain reflection in lowaltitude flight. Such variation results in attenuation of the received signal power. Therefore, statistical models should be used to characterize the received signal in UAV communication. In our case study, we consider a combined model with path loss, shadowing, and fast fading effect. The total path loss with signal attenuation (in $\mathrm{dB}$ ) is given by

$$
Q_{T}=Q_{0}+X+20 \log _{10}(D),
$$

where $X$ represents the log-normal shadowing effect. $X$ (in $\mathrm{dB}$ ) is a zero-mean Gaussian random variable with standard deviation $\sigma$ (in $\mathrm{dB}$ ). For large-scale path loss without the fast fading effect, the cumulative distribution function is given by

$$
\begin{aligned}
\operatorname{Pr}\left(Q_{T} \leq Q_{\text {threshold }}\right) & =\int_{-\infty}^{a} \frac{1}{\sigma \sqrt{2 \pi}} \exp \left(-\frac{X^{2}}{2 \sigma^{2}}\right) d X \\
& =1-\frac{1}{2} \operatorname{erfc}\left(\frac{a}{\sqrt{2} \sigma}\right),
\end{aligned}
$$

where $a=Q_{\text {threshold }}-Q_{0}$. Equation (7) can be used to find the outage probability.

In equation (6), $D$ is a random variable that represents the received envelop of the fast fading signal. It follows Rayleigh distribution or Rician distribution. The probability density function of the Rayleigh distribution is given by

$$
p(D)=\frac{D}{\rho^{2}} \exp \left(-\frac{D^{2}}{2 \rho^{2}}\right), \quad D \geq 0,
$$

where $\rho^{2}$ is the time-average power of the received signal. When there is an unobstructed LOS between the transmitting and receiving UAVs, $D$ follows the Rician distribution, which is given by

$$
p(D)=\frac{D}{\rho^{2}} \exp \left(-\frac{D^{2}+A^{2}}{2 \rho^{2}}\right) I_{0}\left(\frac{A D}{\rho^{2}}\right), \quad D \geq 0,
$$

TABLE 2: Medium access control header.

\begin{tabular}{lccccc}
\hline $\begin{array}{l}\text { Frame } \\
\text { control }\end{array}$ & Duration & Address 1 & Address 2 & Address 3 & $\begin{array}{l}\text { Sequence } \\
\text { control }\end{array}$ \\
\hline ( 2 bytes $)$ & $(2$ bytes $)$ & $(6$ bytes $)$ & $(6$ bytes $)$ & $(6$ bytes $)$ & $(2$ bytes $)$ \\
\hline
\end{tabular}

TABLE 3: Medium access control data unit.

\begin{tabular}{llc}
\hline MAC header & Frame body & FCS \\
\hline (24 bytes $)$ & (2312 bytes) & (4 bytes $)$ \\
\hline
\end{tabular}

TABLE 4: ACK frame.

\begin{tabular}{lccc}
\hline $\begin{array}{l}\text { Frame } \\
\text { control }\end{array}$ & Duration & Receiver address & FCS \\
\hline \begin{tabular}{l} 
(2 bytes $)$ \\
\hline
\end{tabular} & (2 bytes $)$ & (6 bytes $)$ & (4 bytes $)$ \\
\hline
\end{tabular}

TABle 5: Physical layer data frame.

\begin{tabular}{llc}
\hline Preamble & Header & MAC data unit \\
\hline (144 bits $)$ & (48 bits $)$ & xxxx \\
\hline
\end{tabular}

where $A$ denotes the peak amplitude of the dominant (LOS) signal component, and $I_{0}(\cdot)$ is the zero-order modified Bessel function of the first kind

$$
I_{0}(x)=\frac{1}{2 \pi} \int_{-\pi}^{\pi} \exp (-x \sin \tau) d \tau .
$$

The ratio $A^{2} /\left(2 \rho^{2}\right)$ is called the Rician $K$ factor. This parameter $K$ measures the link quality and represents the ratio of the power in the dominant (LOS) component to the power in other non-light-of-sight (NLOS) multipath components. As $K$ increases, the link becomes clearer with less fading. The average received power in the Rician fading can be calculated as

$$
\bar{P}_{r}=\int_{0}^{\infty} D^{2} p(D) d D=A^{2}+2 \rho^{2} .
$$

Substitute $A^{2}=K \bar{P}_{r} /(K+1)$ and $2 \rho^{2}=\bar{P}_{r} /(K+1)$ in equation (9), we can write the Rician distribution in terms of $K$ and $\bar{P}_{r}$ as

$$
\begin{gathered}
p(D)=\frac{2 D(K+1)}{\bar{P}_{r}} \exp \left(-K-\frac{(K+1) D^{2}}{\bar{P}_{r}}\right) \\
\cdot I_{0}\left(2 D \sqrt{\frac{K(K+1)}{\bar{P}_{r}}}\right), \quad D \geq 0 .
\end{gathered}
$$

4.2. Packet Transmission Time. To evaluate communication of the UAV ad hoc network, we analyze the performance of the MAC layer and the physical layer in terms of total time needed to transmit a packet. The time needed to transmit a packet is given by

$$
\begin{aligned}
T_{\text {total }}= & \text { DIFS }+ \text { BackOffTime }+(\text { Data }(\text { bytes }) \\
& +28(\text { bytes })) \times \frac{8}{\text { DataRate }(\text { bit } / \text { sec })}+\text { SIFS } \\
& + \text { OverheadTime }+ \text { ACKTime. }
\end{aligned}
$$


TABLE 6: Simulation parameters.

\begin{tabular}{lc}
\hline Parameter & Value \\
\hline Number of UAVs & 4 \\
Mobility & Hexagon route \\
Simulation time & 60 minutes \\
Data rate & $11 \mathrm{Mbps}$ \\
Area & $2000 \mathrm{~m} \times 2000 \mathrm{~m}$ \\
RTS threshold & $256 \mathrm{bytes}$ \\
$\begin{array}{l}\text { Receive power } \\
\text { threshold }\end{array}$ & $-95 \mathrm{dBm}$ \\
$\begin{array}{l}\text { Transmit power } \\
\text { Packet size }\end{array}$ & $1 \mathrm{~mW}$ \\
$\begin{array}{l}\text { Packet interarrival } \\
\text { time }\end{array}$ & $1024 \mathrm{bits}$ \\
$\begin{array}{l}\text { Destination IP } \\
\text { address }\end{array}$ & Exponential \\
Radio propagation \\
model
\end{tabular}

DIFS and SIFS are used to ensure packet reception and avoid collision between packets. The time specified for DIFS and SIFS differs based on the physical layer. In the following, we consider direct-sequence spread spectrum (DSSS) parameters where SIFS $=10 \mu \mathrm{s}, T_{\text {Slot }}=20 \mu \mathrm{s}$, DIFS $=$ SIFS + $2 \times T_{\text {Slot }}=10+2 \times 20=50 \mu s$, and BackOffTime $=$ $T$ _Slot $\times \operatorname{Random}(\mathrm{CW})=20 \times 31=620 \mu$ s.

The header of the MAC layer is shown in Table 2 and it consists of 24 bytes. The whole data unit is shown in Table 3, where the frame control unit sequence (FCS) is attached to frame body and it has 4 bytes. Thus, 28 bytes compromise the overhead in the MAC layer. The data length is limited to 2312 bytes in 802.11 b and 4095 bytes in $802.11 \mathrm{~g}$. ACK packet is short in size ( 14 bytes) and is shown in Table 4 .

The overhead of the physical layer consists of a preamble and a header. Table 5 shows the physical layer data frame in which a 144-bit preamble and a 48-bit header are added to the frame. Therefore, with a 11-Mbps data rate, the time needed to transmit a $802.11 \mathrm{~b}$ packet is

$$
\begin{aligned}
T_{\text {total }}= & 50+620+(2312+28) \times \frac{8}{11}+10 \\
& +\frac{(144+48)}{11}+14 \times \frac{8}{11} \\
= & 2409.45 \mu \mathrm{s} .
\end{aligned}
$$

\section{Implementation of UAV Ad Hoc Communication Network in OPNET}

5.1. OPNET Modeler. The Optimized Network Engineering Tool (OPNET) modeler is considered a powerful software tool for network modeling and network performance analysis. The OPNET modeler is a discrete-event network simulator that includes a set of detailed models for ad hoc network. It uses graphic user interfaces and allows users to create new models by either modifying existing models or building new one. It uses finite state machine (FSM) model in which a collection of states are linked together based on $\mathrm{C}$ code. Each state is divided into two parts: enter executive and exit executive. Both parts specify a series of actions that a process implements when it occupies a state. The enter executive is executed as soon as the state is entered by the process while the exit executive is used in the unforced state to implement a response to an interrupt.

5.2. Radio Transceiver Pipeline. In our simulations, we modeled the wireless link between the transmitter and the receiver with fourteen pipeline stages. These stages are provided by the OPNET modeler and are connected between the transmitter and the receiver as shown in Figure 3. Six stages are associated with the radio transmitter and eight stages are associated with the radio receiver.

5.3. UAV Mobility Model. The movement of the UAV has a significant influence on the performance of the network. Therefore, a lot of research has been devoted to build different mobility models that are suitable for evaluating the performance of the UAV ad hoc network. A large number of mobility models were introduced. They have different properties and each has its own advantages and disadvantages. In our case study, the mobility model for UAV should be close to real life. We model the mobility of the UAV with six parameters (pitch, roll, yaw, latitude, longitude, and altitude). Each UAV moves in a hexagon route as shown in Figure 4.

5.4. Modeling UAV Network Node with Two Directional Antennas and Two Omnidirectional Antennas. As shown in Figure 5, a UAV network node is modeled according to the Open Systems Interconnection (OSI) stack. Some layers are omitted and some modifications are added to the original modules. The UAV model consists of three main parts: physical layer, data link layer, and upper layer. The bottom part represents the physical layer. This part is slightly different from the OPNET standard model. As shown in the figure, the physical layer is composed of the transmitter module, the receiver module, and the antenna module. In UAV transceiver modeling, we use three transmitters, one receiver, and four antennas. One omnidirectional antenna is connected to the receiver module and three antennas are connected to the transmitter modules. One of the three is an omnidirectional antenna and the other two are directional antennas. All of the above modules are responsible for the wireless communication between UAVs.

The middle part is the data link layer. This part is divided into two modules. The first one is the original MAC module (wireless_lan_mac) and the second one is the proposed module (UAV_SUB_MAC). The wireless_lan_mac module implements the MAC protocol defined by the IEEE 802.11 standards. It is designed mainly to be used with omnidirectional antennas. Some modifications have been made to this module in order to link the directional antennas with the radio transmitter modules and enable this module to work as a two-mode module. The second 


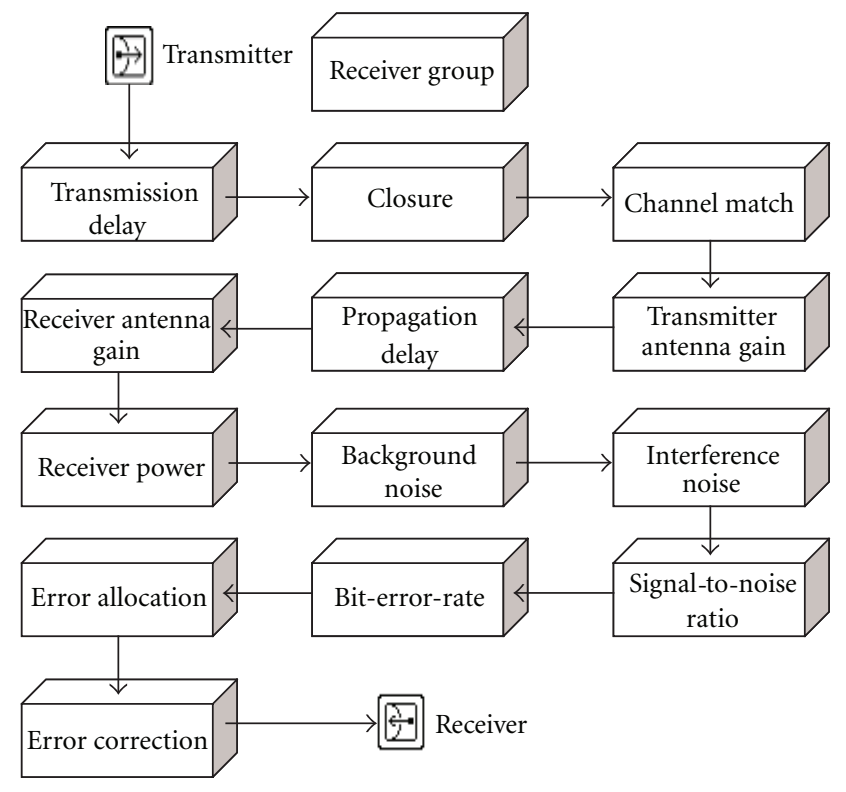

FIGURE 3: OPNET radio transceiver pipeline stages.

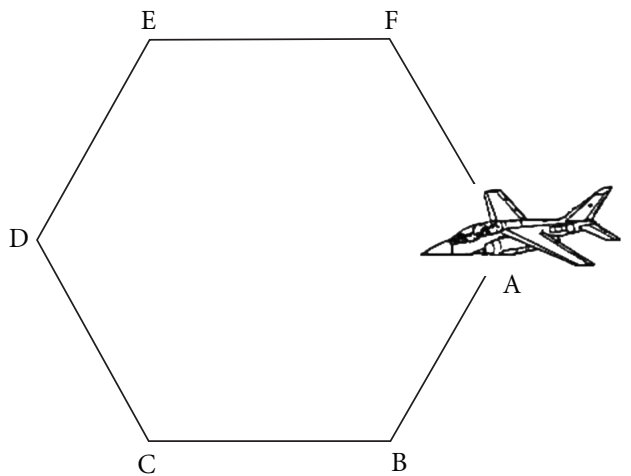

FIgURE 4: UAV mobility model in OPNET.

module implements the protocol described in Section 3, and it acts as an interface between the wireless_lan_mac and the lower layer. Both modules work jointly to serve the proposed directional MAC scheme.

The top part represents the upper layers. The upper layers are mainly composed of the modules such as ARP module, IP module, IP_ENCAP module, TRAF_SRC module, UDP module, DHCP module, MANET_RTE_MGR module, and CPU module. These modules generate data packets and implement the Optimized Link State Routing (OLSR) protocol. For example, TRAF_SRC module performs the function of generating raw packets. These packets are unformatted bits which are encapsulated as IP datagram by the IP_ENCAP module. The IP module implements the IP protocol and the MANET_RTE_MGR module implements the OLSR protocol and manages the statistics for simulation runs.

As discussed earlier, the UAV_SUB_MAC module works jointly with the wireless_lan_mac module. Figure 6 shows the process model for the UAV_SUB_MAC. The process is constructed by seven states. The function of each state is described in the following.

(1) Init State: This state initializes the state variables and the target information table.

(2) Idle State: This is the default state. The node enters the idle state and waits for an incoming event. The event can be either self-interrupt or an incoming packet from the wireless_lan_mac module. An incoming packet from the wireless_lan_mac will be checked based on its type, and control packet will be sent to the Omni State while data packet will be sent to Target_Table State. In addition, this state will read the parameters that affect the selection of antenna as well as the MAC attribute values.

(3) Omni State: In this state, the incoming packet is forwarded to the omnidirectional antenna.

(4) Reset State: This state adds some delay to permit other modules to register themselves.

(5) Targe_Table State: This state determines whether the packet belongs to the primary state or the secondary state based on the altitude of the UAV.

(6) Primary State: In this state, the target location is obtained in order to point the primary directional antenna to that location. The UAV attitude is recorded for each packet so that any change will trigger the compensator.

(7) Secondary State: This state performs the same functionality as the primary state, but uses the secondary directional antenna.

As soon as a packet is received by the wireless_lan_mac module from the upper layer, the wireless_lan_mac module 


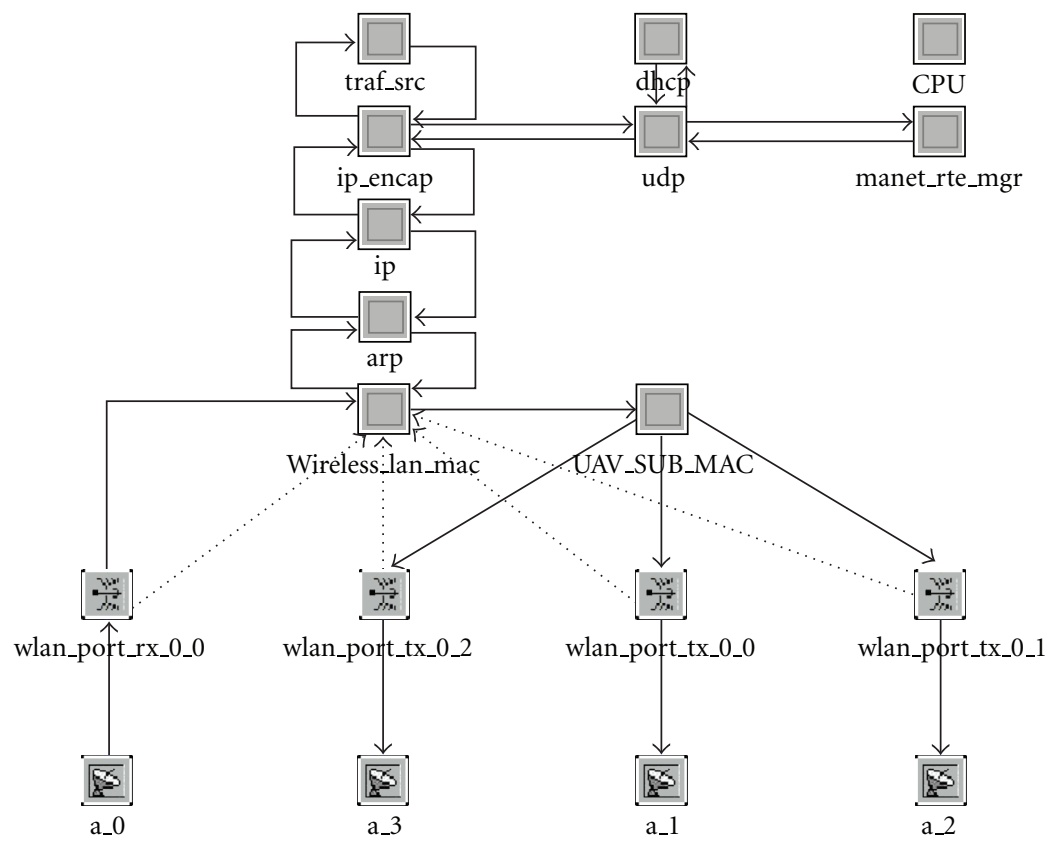

FIgURE 5: Modeling UAV network node in OPNET.

encapsulates the packet into a frame and sends it to the UAV_SUB_MAC module. In the OPNET simulator, each node has an ID. All nodes involved in the network register their IDs in a global array. The UAV_SUB_MAC module (Primary State and Secondary State) fetches the destination node ID from the received packet and retrieves its location from the global array. Since all nodes are mobile, the proposed module fetches the target location for each packet, which includes longitude in degrees, latitude in degrees, and altitude in meters. This information is then used by the Primary State or the Secondary State to point the main lobe of the directional antenna to the target location.

\section{OPNET Simulation Results}

OPNET Modeler 14.5 is used for simulation of the UAV ad hoc communication network. With the parameters shown in Table 6, we compare the network performance of the proposed AMAC_UAV protocol using directional antennas with that of the IEEE 802.11 MAC protocol using omnidirectional antennas. Four UAVs form a MANET in a simulated area of $2000 \mathrm{~m} \times 2000 \mathrm{~m}$. Both AMAC_UAV and IEEE 802.11 MAC protocols operate at a data rate of $11 \mathrm{Mbps}$. The simulation time is 60 minutes, and the UAVs are moving in the simulated area according to the hexagonroute mobility model with a constant speed of $40 \mathrm{~m} / \mathrm{sec}$. The packet size is set to be 1024 bits and the interarrival time is exponentially distributed. All UAV nodes in the network are configured to run the OLSR protocol. Moreover, each UAV is equipped with a transmitter, a receiver, two directional antennas, and two omnidirectional antennas. Each UAV is capable of electronically steering the antenna beam toward a desired direction. For each data packet to be transmitted, the directional antenna is steered based on the destination location as well as the source Euler Angles.

Figure 7 shows the performance comparison in end-toend delay between the AMAC_UAV protocol using directional antenna and the IEEE 802.11 MAC protocol using omnidirectional antenna. The end-to-end delay represents the time interval from the instant that a packet is generated by the source node to the instant that the packet is received by the destination node. This time interval increases when the packet passes through multiple hops between the source and the destination. The figure shows that the AMAC_UAV protocol results in small end-to-end delay compared with the IEEE 802.11 MAC protocol. This is because that the number of hops is reduced when directional antennas are used with extended transmission range.

Figure 8 shows the performance comparison in throughput between the two protocols. The throughput of a communication network is defined as the average rate of successful message delivery. In the figure, the maximum throughput achieved by the IEEE 802.11 MAC protocol with omnidirectional antennas is less than 500 bps over the entire simulation time. It decreases as the UAVs start to move away from each other. With the AMAC_UAV protocol with directional antennas, as the UAVs move away from each other, the throughput increases until reaching a saturation point. The result indicates that the network throughput can be increased by using the AMAC_UAV protocol with directional antennas. Figure 9 supports the above result. It reveals that the maximum traffic received by a particular UAV node using the IEEE 802.11 MAC with omnidirectional antenna is no more than 0.4 packet/sec. For the same amount of traffic sent, the node receives more packets when using the AMAC_UAV protocol with directional antennas. 


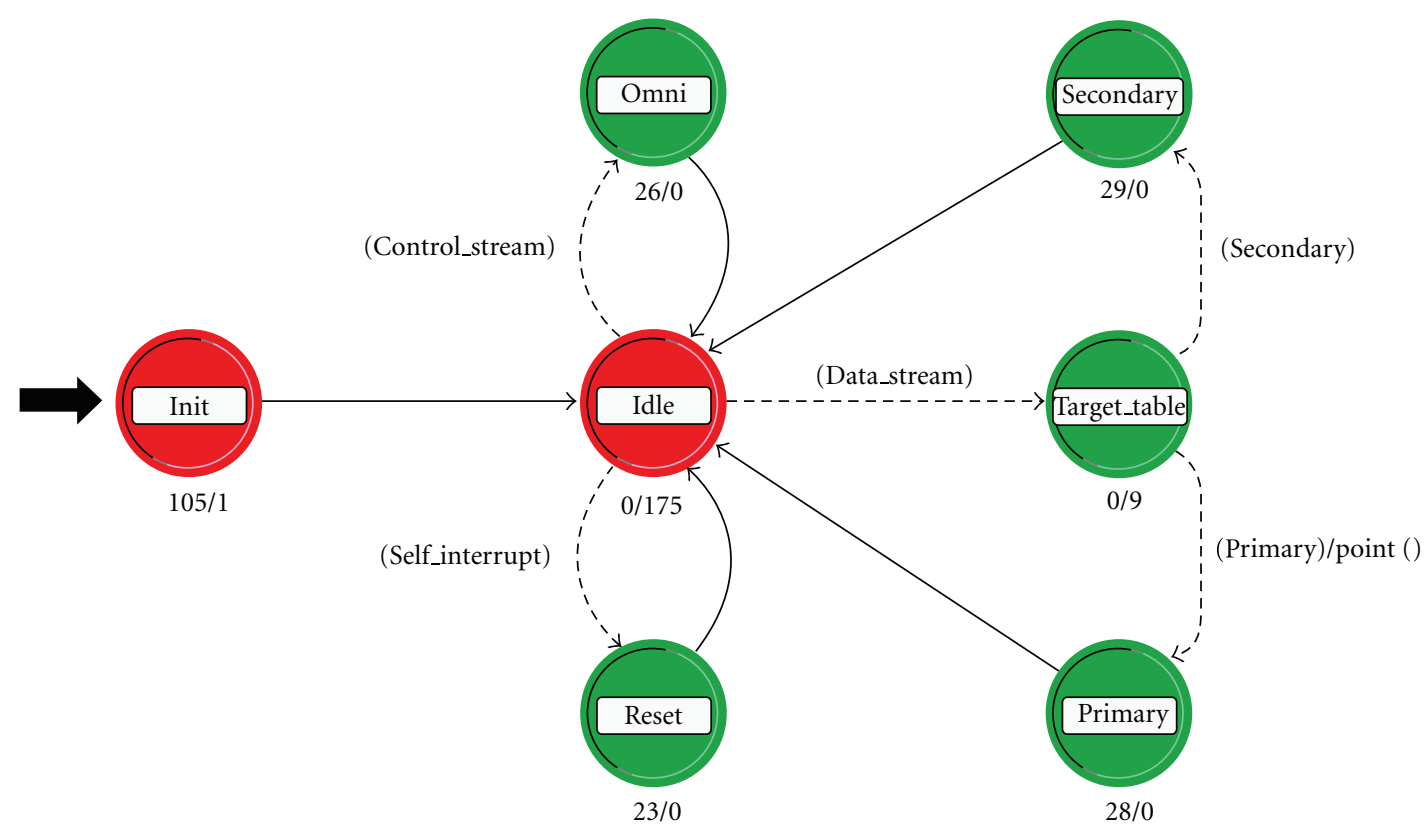

Figure 6: OPNET process model of UAV_SUB_MAC.

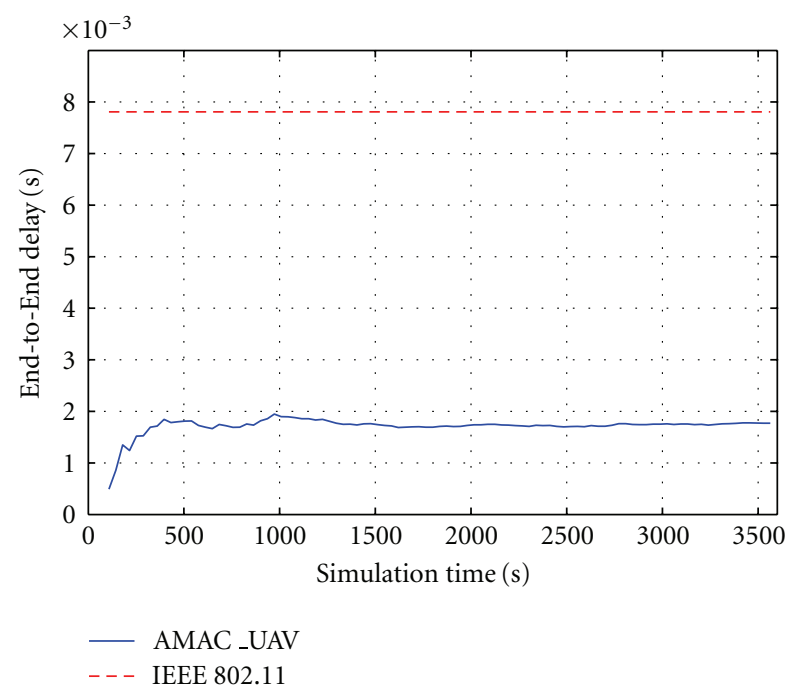

FIgURE 7: End-to-end delay of a MANET of 4 UAVs.

Figure 10 presents the simulation result regarding the receive SNR. The receive SNR gives an indication about the quality of the received signal in which the higher the SNR the better the signal quality. As shown in the figure, the received SNR of the UAV ad hoc communication network employing the AMAC_UAV protocol with directional antennas is considerably higher than that using the IEEE 802.11 MAC protocol. Both protocols are modeled with the same pipelines that compute the background noise and the interference affecting the incoming signal. In the simulation, the SNR result of the AMAC_UAV protocol is ideal, since it is assumed that the steering of the directional antenna is precise and the interference is kept to a minimum.

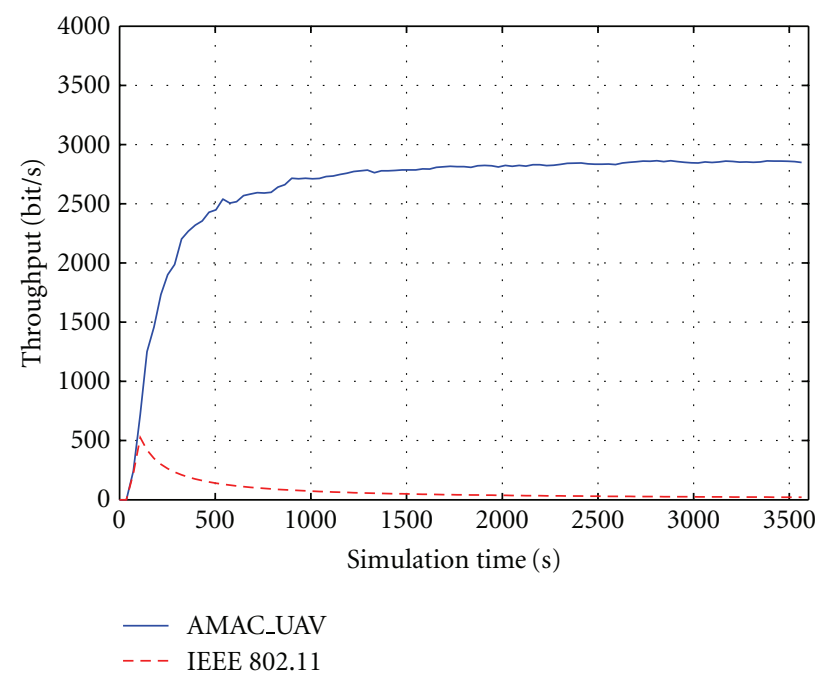

FIgURE 8: Throughput of a MANET of 4 UAVs.

Figure 11 shows the comparison in received BER between the UAV ad hoc communication networks employing the two protocols. The result is consistent with the previous result in received SNR. As shown in the figure, the AMAC_UAV protocol gives less BER than the standard IEEE 802.11 protocol. The proposed protocol gives a zero BER in the first 1750 seconds, while the standard protocol gives more than $3 \times 10^{-5}$ BER over the same period.

In another scenario, we simulate a MANET that is formed by twenty five UAVs. In a simulated area of $2000 \mathrm{~m}$ $\times 2000 \mathrm{~m}$, the UAVs are initially deployed as shown in Figure 12. The simulation time is 10 minutes, and the UAVs are moving in the simulated area according to a random 


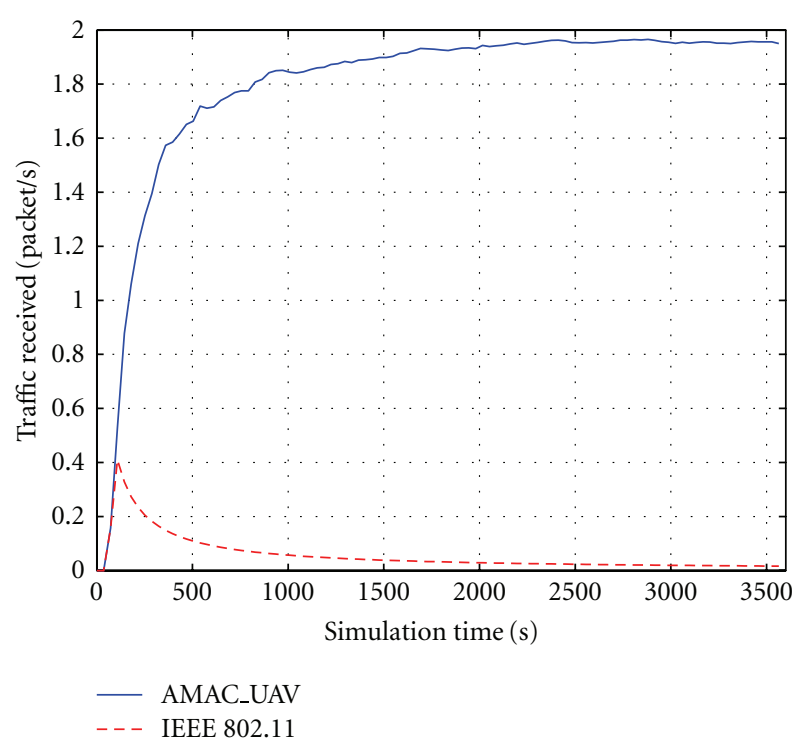

Figure 9: Traffic received of a MANET of 4 UAVs.

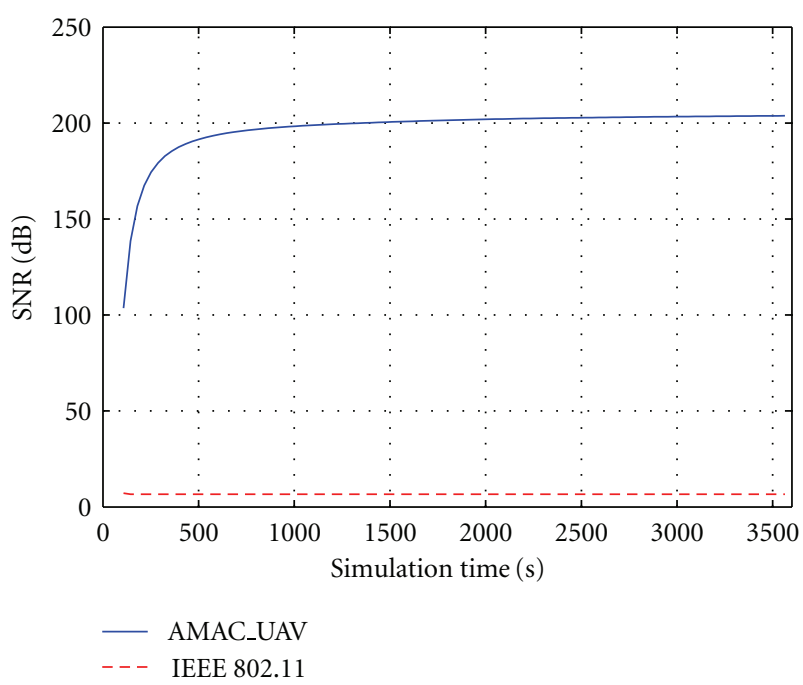

Figure 10: Receive SNR of a MANET of 4 UAVs.

waypoint model with a zero pause time and a constant speed of $40 \mathrm{~m} / \mathrm{sec}$. All UAVs in the network are configured to run the OLSR protocol. The packet size is set to be 1024 bits and the interarrival time is exponentially distributed. The date rate is $11 \mathrm{Mbps}$ and the transmit power level is $1 \mathrm{~mW}$.

Figure 13 shows the performance comparison in end-toend delay of this 25-node network between the AMAC_UAV protocol with directional antennas and the IEEE 802.11 MAC protocol with omnidirectional antennas. In general, there are three factors that affect the end-to-end delay of a packet: time to discover the route, buffering waiting time, and number of hops of each path. Since the number of hops is reduced with the AMAC_UAV protocol, the end-to-end delay decreases. The end-to-end delay with both protocols is high at the beginning of the simulation time. This reflects the fact that

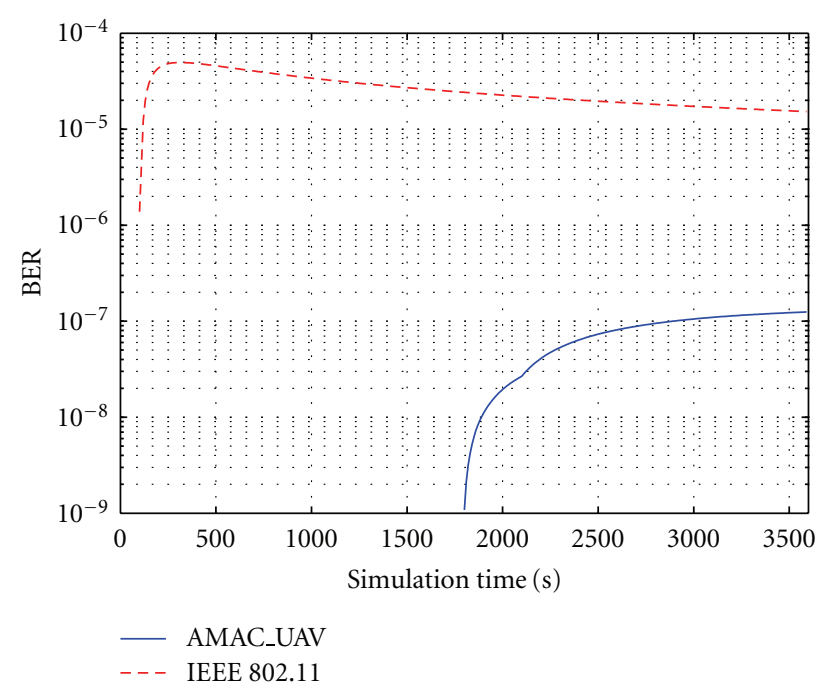

FIgURE 11: Receive BER of a MANET of 4 UAVs.
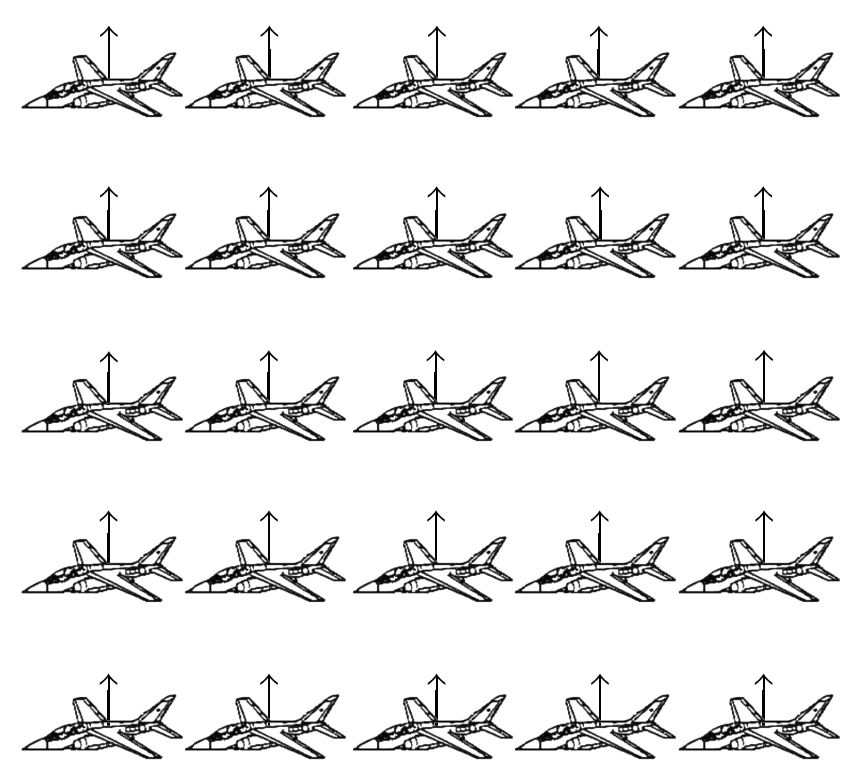

FIgURE 12: Initial topology of a MANET of 25 UAVs.

the size of the control traffic is large before the selection of the Multi-Point Relaying (MPR) set.

\section{Conclusion}

A novel adaptive medium access control protocol is proposed for UAV mobile ad hoc communication networks, which is called AMAC_UAV. Each UAV functions as a network node and is equipped with two directional antennas and two omnidirectional antennas. The UAV is able to receive packet using the omnidirectional antenna and transmit packet using either the directional or the omnidirectional antenna. The transmitter can also choose from the two directional antennas mounted on top of and beneath the UAV and can steer 


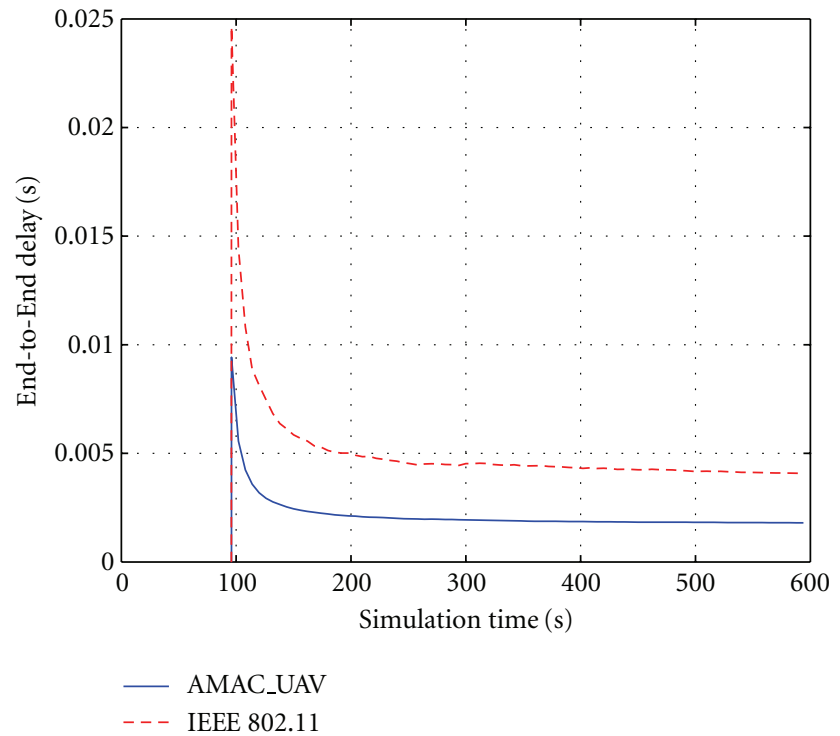

Figure 13: End-to-end delay of a MANET of 25 UAVs.

the antenna beam toward the target. The OPNET Modeler is used to implement the proposed MAC protocol for the UAV ad hoc network. Each UAV network node is modeled and the antenna patterns are constructed by the antenna pattern editor provided by OPNET. The data collected from the simulator are analyzed and compared with the same scenario but using standard IEEE 802.11 MAC protocol with only omnidirectional antennas. It is revealed that the proposed AMAC_UAV scheme with directional antennas provides better performance for UAV ad hoc communication networks in terms of end-to-end delay and throughput.

\section{References}

[1] A. Urpi, M. Bonuccelli, and S. Giordano, "Modeling cooperation in mobile ad hoc networks: a formal description of selfishness," in Proceedings of Conference on Modeling and Optimization in Mobile, Ad Hoc and Wireless Networks (WiOpt '03), March 2003.

[2] I. Rubin and R. Zhang, "Placement of UAVs as communication relays aiding mobile ad hoc wireless networks," in Proceedings of the Military Communications Conference (MILCOM '07), October 2007.

[3] S. Srinivasan, H. Latchman, J. Shea, T. Wong, and J. McNair, "Airborne traffic surveillance systems-video surveillance of highway traffic," in Proceedings of the ACM 2nd International Workshop on Video Surveillance and Sensor Networks (VSSN '04), pp. 131-135, October 2004.

[4] K. Erik and S. Nadjm-Tehrani, "Mobility models for UAV group reconnaissance applications," in Proceedings of the 2nd International Conference on Wireless and Mobile Communications, Bucharest, Romania, July 2006.

[5] D. Hague, H. T. Kung, and B. Suter, "Field experimentation of cots-based UAV networking," in Proceedings of Military Communications Conference (MILCOM '06), October 2006.

[6] M. Takai, J. Martin, A. Ren, and R. Bagrodia, "Directional virtual carrier sensing for directional antennas in mobile ad hoc networks," in Proceedings of the 3rd ACM International
Symposium on Mobile Ad Hoc Networking and Computing (MOBIHOC '02), pp. 183-193, June 2002.

[7] Y. B. Ko, V. Shankarkumar, and N. H. Vaidya, "Medium access control protocols using directional antennas in ad hoc networks," in Proceedings of the 19th Annual Joint Conference of the IEEE Computer and Communications Societies (INFOCOM '00), pp. 13-21, March 2000.

[8] Z. Huang and C. C. Shen, "A comparison study of omnidirectional and directional MAC protocols for ad hoc networks," in Proceedings of the IEEE Global Telecommunications Conference (GLOBECOM '02), pp. 57-61, November 2002.

[9] R. Ramanathan, "On the performance of ad-hoc networks with beam-forming antennas," in Proceedings of the 2nd ACM International Symposium on Mobile Ad-Hoc Networking and Computing, pp. 95-105, Long Beach, Calif, USA, October 2001.

[10] S. Horisawa, S. Bandyopadhyay, K. Hausike, and S. Tawara, "An adaptive MAC and directional routing protocol for adhoc wireless networks using ESPAR antenna," in Proceedings of the 2nd ACM International Symposium on Mobile Ad-Hoc Networking and Computing, pp. 243-246, October 2001.

[11] J. C. Mundarath, P. Ramanathan, and B. D. van Veen, "NULLHOC: a MAC protocol for adaptive antenna array based wireless ad hoc networks in multipath environments," in Proceedings of the IEEE Global Telecommunications Conference (GLOBECOM '04), vol. 5, pp. 2765-2769, Dallas, Tex, USA, November-December 2004.

[12] R. R. Choudhury, X. Yang, R. Ramanathan, and N. H. Vaidya, "On designing MAC protocols for wireless networks using directional antennas," IEEE Transactions on Mobile Computing, vol. 5, no. 5, pp. 477-491, 2006.

[13] A. Singh, P. Ramanathan, and B. van Veen, "Spatial reuse through adaptive interference cancellation in multi-antenna wireless networks," in Proceedings of the IEEE Global Telecommunications Conference (GLOBECOM '05), vol. 5, pp. 30923096, December 2005.

[14] M. Takata, M. Bandai, and T. Watanabe, "Performance analysis of a directional MAC protocol for location information staleness in MANETs," IPSJ Digital Courier, vol. 1, pp. 518527, 2005.

[15] A. Nasipuri, S. Ye, J. You, and R. E. Hiromoto, "A MAC protocol for mobile ad hoc networks using directional antennas," in Proceedings of IEEE Wireless Communications and Networking Conference, vol. 3, pp. 1214-1219, Chicago, Ill, USA, September 2000.

[16] N. S. Fahmy and T. D. Todd, "A selective CSMA protocol with cooperative nulling for ad hoc networks with smart antennas," in Proceedings of IEEE Wireless Communications and Networking Conference (WCNC '04), March 2004.

[17] H. Singh and S. Singh, "Tone based MAC protocol for use with adaptive array antennas," in Proceedings of IEEE Wireless Communications and Networking Conference (WCNC '04), March 2004.

[18] L. Bao and J. J. Garcia-Luna-Aceves, "Transmission scheduling in ad hoc networks with directional antennas," in Proceedings of the 8th Annual International Conference on Mobile Computing and Networking (MobiCom '02), pp. 48-58, September 2002.

[19] M. Takai, J. Martin, A. Ren, and R. Bagrodia, "Directional virtual carrier sensing for directional antennas in mobile ad hoc networks," in Proceedings of the 3rd ACM International Symposium on Mobile Ad Hoc Networking and Computing (MOBIHOC '02), pp. 183-193, June 2002. 
[20] R. R. Choudhury, X. Yang, R. Ramanathan, and N. H. Vaidya, "Using directional antennas for medium access control in ad hoc networks," in Proceedings of the 8th Annual International Conference on Mobile Computing and Networking (MOBICOM '02), pp. 59-70, Atlanta, Ga, USA, September 2002.

[21] R. Ramanathan, J. Redi, C. Santivanez, D. Wiggins, and S. Polit, "Ad hoc networking with directional antennas: a complete system solution," IEEE Journal on Selected Areas in Communications, vol. 23, no. 3, pp. 496-506, 2005.

[22] H. Jasani and K. Yen, "Performance improvement using directional antennas in ad hoc networks," International Journal of Computer Science and Network Security, vol. 6, no. 6, pp. 180$188,2006$.

[23] J. Wang, Y. Fang, and D. Wu, "Syn-dmac: a directional mac protocol for ad hoc networks with synchronization," in Proceedings of IEEE Military Communications Conference, vol. 4, pp. 2258-2263, October 2005.

[24] P. C. Yeh, W. E. Stark, and S. A. Zummo, "Performance analysis of wireless networks with directional antennas," IEEE Transactions on Vehicular Technology, vol. 57, no. 5, pp. 31873199, 2008.

[25] T. Korakis, G. Jakllari, and L. Tassiulas, "A MAC protocol for full exploitation of directional antennas in ad-hoc wireless networks," in Proceedings of the 4th ACM International Symposium on Mobile Ad Hoc Networking and Computing (MOBIHOC '03), pp. 98-107, June 2003.

[26] G. Jakllari, W. Luo, and S. V. Krishnamurthy, "An integrated neighbor discovery and MAC protocol for ad hoc networks using directional antennas," IEEE Transactions on Wireless Communications, vol. 6, no. 3, pp. 1114-1124, 2007.

[27] R. R. Choudhury and N. H. Vaidya, "Deafness: a MAC problem in ad hoc networks when using directional antennas," in Proceedings of the 12th IEEE International Conference on Network Protocols (ICNP '04), pp. 283-292, October 2004.

[28] J. Yang, J. Li, and M. Sheng, "MAC protocol for mobile ad hoc network with smart antennas," Electronics Letters, vol. 39, no. 6, pp. 555-557, 2003.

[29] T. X. Brown, B. Argrow, C. Dixon, S. Doshi, R. G. Thekkekunel, and D. Henkel, "Ad hoc UAV ground network (AUGNet)," in Proceedings of the 3rd AIAA Unmanned Unlimited Technical Conference, pp. 29-39, Chicago, Ill, USA, September 2004.

[30] T. X. Brown, S. Doshit, S. Jadhav, and J. Himmelstein, "Test bed for a wireless network on small UAVs," in Proceedings of the 3rd AIAA Unmanned Unlimited Technical Conference, pp. 432-439, Chicago, Ill, USA, September 2004.

[31] C.-M. Cheng, P.-H. Hsiao, H. T. Kung, and D. Vlah, "Performance measurement of 802.11a wireless links from UAV to ground nodes with various antenna orientations," in Proceedings of the 15th International Conference on Computer Communications and Networks (ICCCN '06), pp. 303-308, Arlington, Va, USA, October 2006.

[32] R. C. Palat, A. Annamalai, and J. H. Reed, "Cooperative relaying for ad-hoc ground networks using swarm UAVs," in Proceedings of Military Communications Conference (MILCOM '05), vol. 3, pp. 1588-1594, Atlatnic City, NJ, USA, October 2005.

[33] C. M. Cheng, P. H. Hsiao, H. T. Kung, and D. Vlah, "Maximizing throughput of UAV-relaying networks with the loadcarry-and-deliver paradigm," in Proceedings of IEEE Wireless Communications and Networking Conference (WCNC '07), pp. 4420-4427, March 2007. 

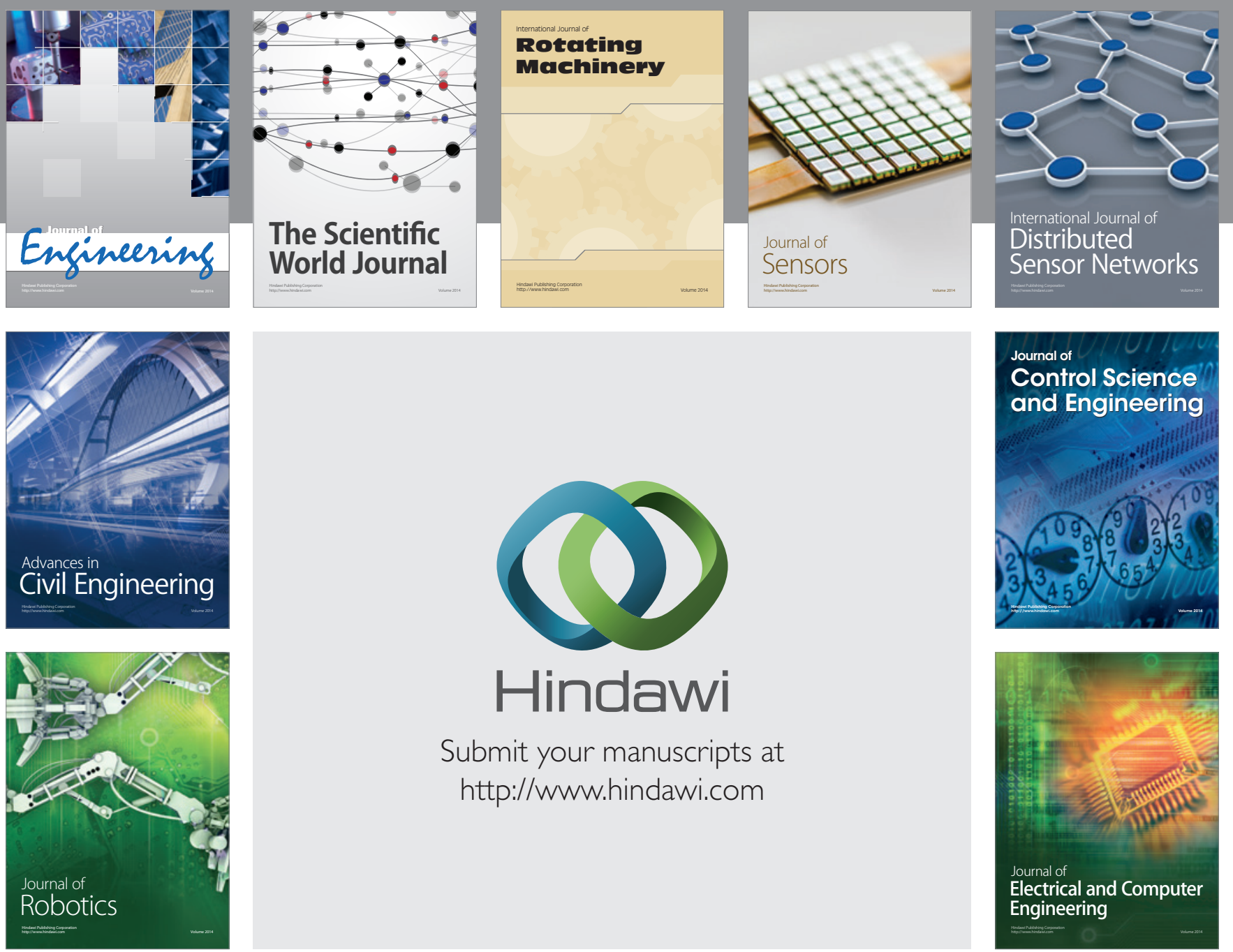

Submit your manuscripts at

http://www.hindawi.com
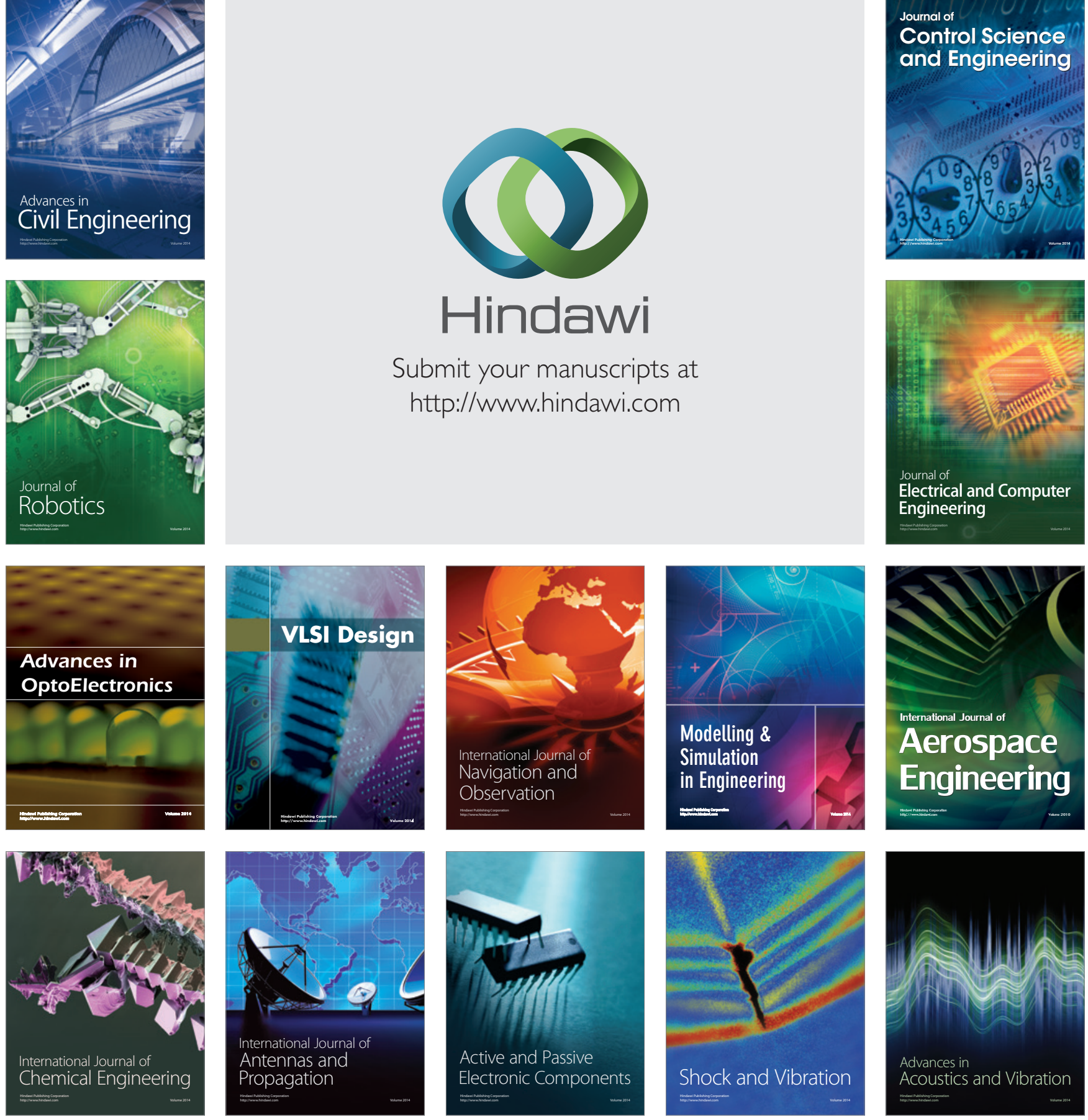